On two diffusion neuronal models with multiplicative noise: The mean first-passage time properties

G. D'Onofrio, P. Lansky, and E. Pirozzi

Citation: Chaos 28, 043103 (2018); doi: 10.1063/1.5009574

View online: https://doi.org/10.1063/1.5009574

View Table of Contents: http://aip.scitation.org/toc/cha/28/4

Published by the American Institute of Physics 


\title{
On two diffusion neuronal models with multiplicative noise: The mean first-passage time properties
}

\author{
G. D’Onofrio, ${ }^{1, a)}$ P. Lansky, ${ }^{1, b)}$ and E. Pirozzi ${ }^{2, c)}$ \\ ${ }^{1}$ Institute of Physiology, Czech Academy of Sciences, Videnska 1083, 14220 Prague 4, Czech Republic \\ ${ }^{2}$ Dipartimento di Matematica e Applicazioni, University of Napoli Federico II, Via Cintia, 80126 Napoli, Italy
}

(Received 17 October 2017; accepted 26 March 2018; published online 5 April 2018)

\begin{abstract}
Two diffusion processes with multiplicative noise, able to model the changes in the neuronal membrane depolarization between two consecutive spikes of a single neuron, are considered and compared. The processes have the same deterministic part but different stochastic components. The differences in the state-dependent variabilities, their asymptotic distributions, and the properties of the first-passage time across a constant threshold are investigated. Closed form expressions for the mean of the first-passage time of both processes are derived and applied to determine the role played by the parameters involved in the model. It is shown that for some values of the input parameters, the higher variability, given by the second moment, does not imply shorter mean firstpassage time. The reason for that can be found in the complete shape of the stationary distribution of the two processes. Applications outside neuroscience are also mentioned. Published by AIP Publishing. https://doi.org/10.1063/1.5009574
\end{abstract}

The first-passage time (FPT) of a stochastic process through a threshold is a problem with direct application in the modeling of neural encoding of information. The membrane depolarization of a neuron fluctuates in response to synaptic inputs and noise. As soon as the depolarization reaches a certain firing threshold, due to chemical reactions taking place in the neuronal membrane, the neuron generates an action potential (spike). This dynamics of spike generation involves the firstpassage-time (or first-hitting-time) and underlies neural coding: the information transferred within the nervous system is encoded by the timing of the spikes. After the generation of the action potential, the voltage is immediately reset and the process starts anew, producing a nonlinearity in the dynamics. Among the classical models, the diffusion leaky integrate-and-fire characterizes a single neuron by a stochastic differential equation describing the evolution in time of the neuronal membrane depolarization. The equation has a linear deterministic part and a stochastic component (an additive noise) expressing the sources of noise. The original models obtained as diffusion approximations of discontinuous models have been modified, introducing a multiplicative noise in the equation, in order to get a better physiological insight. Two of these modified models are considered and compared here using classical and new developed mathematical tools, focusing on the behavior of the depolarization itself and on the random variable first-passage time through the firing threshold. Computationally easy expressions of the first moment of the first-passage time for the two involved processes are derived and implemented.

\section{INTRODUCTION}

The role of noise in information transfer within the nervous system has been one of the commonly approached phenomena during the last several decades. Various neuronal systems as well as their models served as the primary example of the stochastic resonance or related effects. ${ }^{1-4}$ Noiseinduced activities of different types have been investigated on the neuronal models or their networks of various complexities ranging from simple integrators ${ }^{5-9}$ to the full Hodgkin-Huxley schema. ${ }^{10-14}$ All such endeavors confirm that the noise, which in the nervous system is an integral part of the signal, ${ }^{15-18}$ is primarily important, especially for weak, subthreshold signals. While there is an ongoing effort to incorporate detailed biological properties into realistic computer models and simulations, on the other hand, a compromise is usually made and the richness of details is sacrificed for computational or even analytical accessibility. Our investigation presented in this article aims to contribute to this effort in clarifying the role of noise, specifically of the multiplicative noise, on the performance of two simple neuronal models using the analytical approach.

Stochastic diffusion processes have been extensively used to model the changes in the membrane depolarization between two consecutive neuronal spikes (action potentials). Furthermore, by solving the related first-passage-time (FPT) problem, the dynamics of the spike generation has been described. ${ }^{19,20}$ Among all these models, the OrnsteinUhlenbeck process plays a prominent role being the most extensively studied. ${ }^{21,22}$ It is characterized by a linear deterministic part and an additive white noise. It can be obtained as a diffusion approximation of the Stein's model which poses interpretable parameters which property is partly lost during the process of approximation. ${ }^{23}$ This lack of transparency of the parameters in the Ornstein-Uhlenbeck model is compensated by far easier mathematical tractability

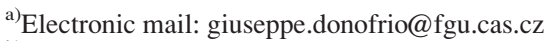

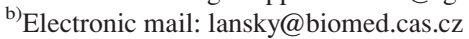

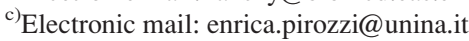


compared to the original Stein's model. The model was shown to fit a number of experimentally recorded neuronal data although some care must be taken. ${ }^{24-26}$

As noticed already in the early days of stochastic neuronal modeling, ${ }^{27,28}$ the Ornstein-Uhlenbeck model as well as its non-continuous counterpart exhibits at least two undesirable features. First, their state space is unlimited, i.e., arbitrary large hyperpolarization values are possible. Second, it is a well known fact that the changes in the depolarization of a nerve cell depend on its actual value which feature is neglected in the models. For this reason, several modifications of the Stein's model which includes a more realistic description of the synaptic transmission were proposed. Finally, alternative models in which the changes in depolarization are state-dependent were suggested as their diffusion approximation. ${ }^{7,29-33}$ The limited state space of the model is a natural consequence of the state-dependency of the input causing depolarizations. ${ }^{34}$ Again, all the diffusion variants keep the linear deterministic part; however, the additive noise is replaced by a multiplicative one. In some biophysical models, multiplicative noise emerges from the ion channel fluctuations that are sources of intrinsic noise and depend on the state of the membrane voltage, ${ }^{35-37}$ although this approach is not considered in this work. Here, we consider two variants of the diffusion models with multiplicative noise and compare them. They differ in the shape of variability profiles in dependency on the actual values of the membrane depolarization. The comparison is performed not only with respect to the behavior of the depolarization itself but first of all with respect to the properties of the first-passage time across a constant threshold. As mentioned, this quantity is identified with the interspike interval in the dynamics of neuronal firing and the importance of the interspike intervals follows as a consequence of the generally accepted hypothesis that the information transferred within the nervous system is encoded by the timing of the spikes. Our comparison is restricted on the first moment of the first-passage times with possible implications on frequency coding.

Both diffusion models investigated here have, in accordance with the conditions imposed on their parameters, a lower non-attainable boundary. The boundary differs from zero due to the physiological applications and interpretations and all the formulas given in the paper contain its value explicitly. The same diffusion processes have several other applications but commonly the lower boundary for them is set to zero. Obtaining new results within the other fields based on the current paper is therefore straightforward.

The first model is probably the most common one in this class, generally denoted as a square-root process. ${ }^{38}$ Due to the historical reasons it is also called, in biological context, the Feller model, ${ }^{39-41}$ but in the mathematical finance is known under the name of Cox-Ingersoll-Ross model. ${ }^{42,43}$ It is also used in survival analysis, ${ }^{44}$ in the modeling of nitrous oxide emission from soil, ${ }^{45}$ and in other applications in physics and computer science. ${ }^{46}$ The second model is closely related to the Geometric Brownian motion. In the interest rate field, it is called the Brennan-Schwartz model, ${ }^{43,47}$ denoted as the GARCH model when used for stochastic volatility and for energy markets, ${ }^{48}$ as the Lognormal diffusion process with exogenous factors when used for forecasting and analysis of growth, ${ }^{49}$ and in real option literature it goes under the names of Geometric Brownian motion with affine drift, ${ }^{50}$ Geometric Ornstein-Uhlenbeck, ${ }^{51}$ or mean reverting Geometric Brownian motion. ${ }^{52}$ Here, following Refs. 53 and 54, we call it Inhomogeneous Geometric Brownian Motion (IGBM).

This paper is organized as follows. In the first part, we introduce the models underlining the common features of the involved stochastic processes and the mathematical tools necessary for their analysis. Furthermore, we recall and develop results on the two stochastic processes under consideration. Particular attention is given to results on the IGBM process that are rare and fragmentary in the literature. Section III is devoted to the comparison of the models, mainly studying the influence of different multiplicative noise on the asymptotic variability and on the first moment of the first-passage time. Comments on the obtained results are given in Sec. IV.

\section{DIFFUSION NEURONAL MODELS WITH INHIBITORY REVERSAL POTENTIALS}

The diffusion leaky integrate-and-fire model is described by an Itô stochastic differential equation of the following type:

$$
d Y_{t}=\left(-\frac{Y_{t}}{\theta}+\mu\right) d t+a\left(Y_{t}\right) d W_{t}, \quad Y_{0}=y_{0},
$$

where $\mu$ characterizes the neuronal input, $\theta$ is called membrane time constant and takes into account the spontaneous voltage decay towards the resting potential (assumed equal to zero here) in the absence of input, the diffusion coefficient $a\left(Y_{t}\right)$ determines the amplitude of the noise, $W=\left\{W_{t}\right\}_{t>0}$ is a standard Wiener process, and $y_{0}$ is the starting depolarization. We note that for $a\left(Y_{t}\right) \equiv \sigma$ constant, the solution of Eq. (1) is an Ornstein-Uhlenbeck process.

In order to introduce inhibitory reversal potential $V_{I}$ $<y_{0}$ in this class of models, we consider model (1) such that the function $a(\cdot)$ depends on the process itself and on $V_{I}$. In particular, we choose $a(\cdot)$ such that $a\left(V_{I}\right)=0$ to ensure that the process cannot take values below $V_{I}$. The behavior of a diffusion process near the endpoints of its state space is determined according to the Feller's classification of boundaries. $^{21,22} \mathrm{We}$ say, that $V_{I}$ is an entrance boundary if $Y_{t}$ cannot reach $V_{I}$ in finite time and there is no probability flow to the outside of the interval $\left[V_{I},+\infty\right)$ or is an exit boundary if the process can attain the value $V_{I}$ but cannot return to the interior of $\left[V_{I},+\infty\right)$. The latter situation is not suitable for our modeling purpose and is not considered in the following.

An alternative method to the description of diffusion process (1) consists of the evaluation of the transition probability density function $f(y, t \mid z, \tau)=\frac{\partial}{\partial y} \mathbb{P}(Y(t)<y \mid Y(\tau)=z)$. It is solution of the Fokker-Planck equation

$$
\begin{aligned}
\frac{\partial f\left(y, t \mid y_{0}, 0\right)}{\partial t}= & -\frac{\partial}{\partial y}\left(-\frac{y}{\theta}+\mu\right) f\left(y, t \mid y_{0}, 0\right) \\
& +\frac{1}{2} \frac{\partial^{2}}{\partial y^{2}} a^{2}(y) f\left(y, t \mid y_{0}, 0\right),
\end{aligned}
$$


with zero-flux boundary condition at $y=V_{I}$ (we want $V_{I}$ to be entrance boundary),

$$
\lim _{y \rightarrow V_{I}^{+}}\left(\left(-\frac{y}{\theta}+\mu\right) f\left(y, t \mid y_{0}, 0\right)-\frac{\partial}{\partial y} a^{2}(y) f\left(y, t \mid y_{0}, 0\right)\right)=0
$$

and initial condition

$$
\lim _{t \rightarrow 0} f\left(y, t \mid y_{0}, 0\right)=\delta\left(y-y_{0}\right) .
$$

If the limit of the transition density $f\left(y, t \mid y_{0}, 0\right)$ as $t \rightarrow \infty$ exists independent of $y_{0}$, we say, that the process $Y_{t}$ admits a stationary distribution.

In most cases, the solution of Eq. (2) is non-trivial but at least some information about the process can be obtained from its moments. The transient and the asymptotic means of $Y_{t}$ are

$$
\begin{gathered}
\mathbb{E}\left[Y_{t} \mid Y_{0}=y_{0}\right]=\mu \theta+\left(y_{0}-\mu \theta\right) e^{-t / \theta}, \\
\lim _{t \rightarrow+\infty} \mathbb{E}\left[Y_{t} \mid Y_{0}=y_{0}\right]:=\mathbb{E}[Y]=\mu \theta
\end{gathered}
$$

and the higher moments of $Y_{t}$ depend on the function $a\left(Y_{t}\right)$ in Eq. (1).

Equation (1) describes the membrane depolarization until the occurrence of an action potential (spike). In accordance with the model, the spikes are generated when the process $Y_{t}$ crosses a voltage threshold $S$ for the first time, so called first-passage time (FPT). In the following, we consider $S$ to be a constant, $S>y_{0}$. The process is reset to the starting point $y_{0}$ after the spike and the evolution starts anew. This reset condition guarantees that the interspike intervals are independent and identically distributed, denoted here by $T$ defined as

$$
T:=\inf \left\{t \geq 0: Y_{t} \geq S \mid y_{0}<S\right\},
$$

with a probability density function $g(t):=g\left(t \mid y_{0}\right)$. The moments of $T$ can be calculated through its Laplace transform $g^{*}(\beta)=\int_{0}^{\infty} e^{-\beta t} g(t) d t$ with $\beta>0$. If we denote by $\mathbb{P}\left(T \mid y_{0}\right)$ $=\int_{0}^{\infty} g(t) d t$ the probability of crossing the threshold $S$ and by $\mathbb{E}[T]$ the mean of FPT, we have the following relations:

$$
\mathbb{P}\left(T \mid y_{0}\right)=\left.g^{*}(\beta)\right|_{\beta=0}, \quad \mathbb{E}[T]=-\left.\frac{d g^{*}(\beta)}{d \beta}\right|_{\beta=0} .
$$

The distribution of $T$ is often unavailable and the definition of $g^{*}(\beta)$ cannot be applied directly, but there exists Siegert's equation for the Laplace transform of $T$

$$
\frac{1}{2} a^{2}\left(y_{0}\right) \frac{\partial^{2} g^{*}(\beta)}{\partial y_{0}^{2}}-\left(\frac{y_{0}}{\theta}-\mu\right) \frac{\partial g^{*}(\beta)}{\partial y_{0}}-\beta g^{*}(\beta)=0,
$$

with initial conditions $g^{*}(\beta)=1$ if $y_{0} \equiv S$ and $g^{*}(\beta)<+\infty$ for any $y_{0}$.

Two possible firing regimes can be considered for the process $Y_{t}$. If the asymptotic mean depolarization given by Eq. (6) is larger than the firing threshold $S$ we say that $Y_{t}$ is in the suprathreshold regime and the firing activity is relatively regular. When $\mu \theta<S$, we say that $Y_{t}$ is in the subthreshold regime and the contribution of the noise to the firing is crucial.

\section{A. The Feller process}

The first model investigated here is given by Eq. (1) with

$$
a\left(Y_{t}\right)=\sigma_{F} \sqrt{Y_{t}-V_{I}} .
$$

Let $k:=\frac{2}{\sigma_{F}^{2}}\left(\mu-\frac{V_{I}}{\theta}\right)$, then $V_{I}$ is an entrance boundary if $k \geq 1$; otherwise, it is attainable and so it is not considered here.

The conditional mean of $Y_{t}$ is given by Eq. (5), the transient and asymptotic variances are

$$
\begin{gathered}
\operatorname{Var}\left[Y_{t} \mid y_{0}\right]=\frac{1}{2} \theta \sigma_{F}^{2}\left(\mu \theta-V_{I}\right)\left(1-e^{-\frac{t}{\theta}}\right)^{2} \\
+\left(y_{0}-V_{I}\right) \theta \sigma_{F}^{2}\left(1-e^{-\frac{t}{\theta}} e^{-\frac{t}{\theta}}\right. \\
\operatorname{Var}_{F}[Y]=\frac{1}{2} \theta \sigma_{F}^{2}\left(\mu \theta-V_{I}\right) .
\end{gathered}
$$

The stationary distribution of the Feller process in the absence of a threshold is a shifted gamma distribution with the following shape, scale, and location parameters

$$
Y \sim \operatorname{Gamma}\left(k, \frac{1}{2} \theta \sigma_{F}^{2}, V_{I}\right) .
$$

The Laplace transform of $T$ in the case of model (1) with $a\left(Y_{t}\right)$ as in Eq. (10), is

$$
g^{*}(\beta)=\frac{\Phi\left(\beta \theta, k, \frac{2\left(y_{0}-V_{I}\right)}{\theta \sigma_{F}^{2}}\right)}{\Phi\left(\beta \theta, k, \frac{2\left(S-V_{I}\right)}{\theta \sigma_{F}^{2}}\right)},
$$

where $\Phi$ is the confluent hypergeometric function of the first kind (or Kummer's function $\left.{ }^{55}\right) \Phi(a, b, z)={ }_{1} F_{1}(a ; b ; z)$, given in terms of the generalized hypergeometric function ${ }_{p} F_{q}$

$$
{ }_{p} F_{q}\left(a_{1}, \ldots, a_{p} ; b_{1}, \ldots, b_{q} ; z\right):=\sum_{n=0}^{\infty} \frac{\left(a_{1}\right)_{n} \cdots\left(a_{p}\right)_{n}}{\left(b_{1}\right)_{n} \cdots\left(b_{q}\right)_{n}} \frac{z^{n}}{n !}
$$

with $(a)_{n}$ the rising factorial defined by $(a)_{n}=a(a+1) \cdots$ $(a+n-1)$ for $n \in \mathbb{N},(a)_{0}=1$. The expressions of the variance (11), the stationary distribution (13), and the Laplace transform of $T$ (14), in the case $V_{I}=0$, can be found in Refs. 22 and 40 and generalization for $V_{I} \neq 0$ is straightforward.

The probability of crossing the threshold $S$ is equal to 1 using Eqs. (8) and (14). The mean of $T$ was calculated in Ref. 56 giving

$$
\begin{aligned}
\mathbb{E}_{F}[T]= & \theta \frac{\left(S-y_{0}\right)}{\mu \theta-V_{I}}+\theta \sum_{n=2}^{\infty} \frac{k^{n} \Gamma(k)}{n \Gamma(k+n)} \\
& \times \frac{\left[\left(S-V_{I}\right)^{n}-\left(y_{0}-V_{I}\right)^{n}\right]}{\left(\mu \theta-V_{I}\right)^{n}},
\end{aligned}
$$

where $\Gamma(z)=\int_{0}^{\infty} x^{z-1} e^{-x} d x$ is the gamma function. 
The convergence of the series on the right-hand side of Eq. (16) is fast in the suprathreshold regime (see Fig. 1 for $\mu>2$ ). Conversely, in the subthreshold regime the sum depends strongly on the number of terms taken into consideration and this affects the results when the formula is implemented numerically. Therefore, an alternative formula for the mean FPT is derived (Appendix A)

$$
\begin{aligned}
\mathbb{E}_{F}[T]= & \theta \frac{c}{b}\left[\left(S-V_{I}\right)_{2} F_{2}\left(1,1 ; 2, b+1 ; c\left(S-V_{I}\right)\right)\right. \\
& \left.-\left(y_{0}-V_{I}\right)_{2} F_{2}\left(1,1 ; 2, b+1 ; c\left(y_{0}-V_{I}\right)\right)\right],
\end{aligned}
$$

where

$$
b=\frac{1}{2 \sigma_{F}^{2}}\left(\mu-\frac{V_{I}}{\theta}\right)=\frac{k}{4}, \quad c=\frac{1}{2 \theta \sigma_{F}^{2}} .
$$

The relative difference between the formulas (16) and (17) when the series in Eq. (16) is replaced by a sum of finite number $n$ of terms is shown in Fig. 1. For increasing $n$, the sum converges to the value given by Eq. (17), but for small $\mu$ the distance is still considerable. Furthermore, we use Eq. (17) that is easy to implement with the package hypergeo for the software R. ${ }^{57}$

\section{B. The inhomogeneous geometric Brownian motion}

The second model investigated here is given by Eq. (1) with

$$
a\left(Y_{t}\right)=\sigma_{G}\left(Y_{t}-V_{I}\right)
$$

If $\mu>V_{I} / \theta$, then $V_{I}$ is an entrance boundary; otherwise, it is an exit one and it is not considered here (cf. Ref. 50 for $V_{I}=0$ ).

The process $Y_{t}$ has the conditional mean given by Eq. (5) and the conditional variance

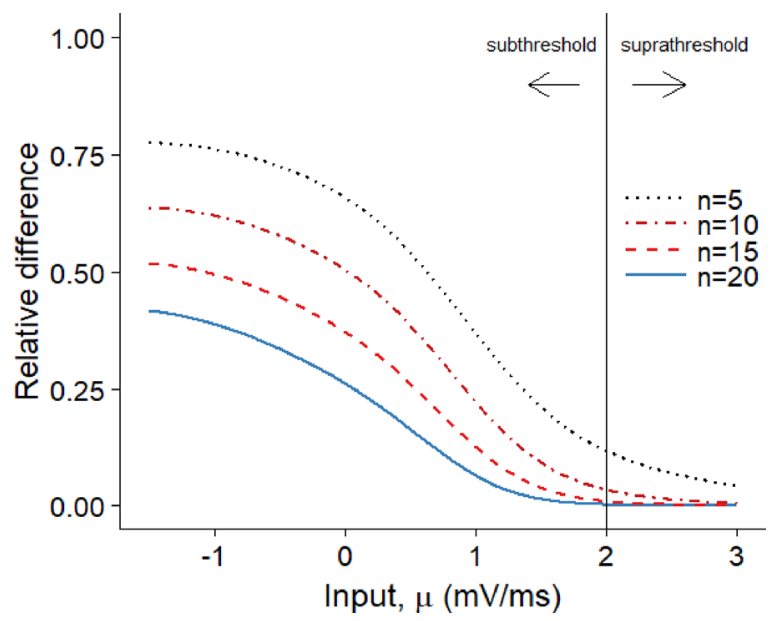

FIG. 1. Relative difference between the formulas (16) and (17) in dependency of $\mu$ for $n$ equal to $5,10,15$, and $20, y_{0}=0 \mathrm{mV}, V_{I}=-10 \mathrm{mV}$, $S=10 \mathrm{mV}, \sigma_{F}=1 / \sqrt{10} \mathrm{mV} / \sqrt{\mathrm{ms}}$, and $\theta=5 \mathrm{~ms}$. For increasing $n$, the sum in Eq. (16) converges to the value given by Eq. (17), but for $n=20$ and $\mu=-1.5 \mathrm{mV} / \mathrm{ms}$ the difference is still almost $50 \%$. The vertical line separates the subthreshold $(\mu<2)$ and suprathreshold regimes $(\mu>2)$ for $S=10 \mathrm{mV}$.

$$
\begin{aligned}
\operatorname{Var}\left[Y_{t} \mid y_{0}\right]= & \frac{\theta \sigma_{G}^{2}\left(\mu \theta-V_{I}\right)^{2}}{2-\theta \sigma_{G}^{2}}+2 \theta \sigma_{G}^{2} \frac{\left(y_{0}-\mu \theta\right)\left(\mu \theta-V_{I}\right)}{1-\theta \sigma_{G}^{2}} e^{-\frac{t}{\theta}} \\
& -e^{-\frac{2 t}{\theta}}\left(y_{0}-\mu \theta\right)^{2}+e^{\left(\sigma_{G}^{2}-\frac{2}{\theta}\right) t} \\
& \times\left[\left(y_{0}-V_{I}\right)^{2}-2 \frac{\left(y_{0}-V_{I}\right)\left(\mu \theta-V_{I}\right)}{1-\theta \sigma_{G}^{2}}\right. \\
& \left.+2 \frac{\left(\mu \theta-V_{I}\right)^{2}}{\left(2-\theta \sigma_{G}^{2}\right)\left(1-\theta \sigma_{G}^{2}\right)}\right] .
\end{aligned}
$$

Equation (19) must be understood as taking the proper limits for $\theta \sigma_{G}^{2}=1$ and $\theta \sigma_{G}^{2}=2$. If $\sigma_{G}^{2}<2 / \theta$, the asymptotic variance is

$$
\operatorname{Var}_{G}[Y]=\frac{1}{2-\theta \sigma_{G}^{2}} \theta \sigma_{G}^{2}\left(\mu \theta-V_{I}\right)^{2}
$$

otherwise, $\operatorname{Var}_{G}[Y]=+\infty$, the case that is not considered here. The above quantities for $V_{I}=0$ are given in Ref. 48, again the generalization for $V_{I} \neq 0$ is straightforward.

The stationary distribution of the IGBM process in the absence of a threshold is a shifted inverse gamma distribution with the following shape, scale, and location parameters

$$
Y \sim \text { Inv-Gamma }\left(1+\frac{2}{\theta \sigma_{G}^{2}}, \frac{2\left(\mu \theta-V_{I}\right)}{\theta \sigma_{G}^{2}}, V_{I}\right) .
$$

The expression of the stationary distribution in the case $V_{I}=0$ is given in other papers although misprints on the scale parameter ${ }^{48}$ or on the form of the distribution ${ }^{50}$ appear.

Generalizing the result of Ref. 54, we obtained the following expression for the Laplace transform of $T$ (see Appendix B)

$$
g^{*}(\beta)=\left(\frac{y_{0}-V_{I}}{S-V_{I}}\right)^{-a} \frac{\Psi\left(a, b ; \frac{c}{y_{0}-V_{I}}\right)}{\Psi\left(a, b ; \frac{c}{S-V_{I}}\right)},
$$

where

$$
\begin{aligned}
& a=\frac{\sqrt{\sigma_{G}^{4}+4\left(\frac{1}{\theta}+2 \beta\right) \sigma_{G}^{2}+\frac{4}{\theta^{2}}}-\left(\frac{2}{\theta}+\sigma_{G}^{2}\right)}{2 \sigma_{G}^{2}} \\
& b=\frac{2}{\theta \sigma_{G}^{2}}+2 a+2 ; \quad c=\frac{2}{\sigma_{G}^{2}}\left(\mu-\frac{V_{I}}{\theta}\right)
\end{aligned}
$$

and $\Psi$ is the confluent hypergeometric function of the second kind (or Tricomi's function ${ }^{55}$ )

$$
\Psi(a, b, z)=z^{-a}{ }_{2} F_{0}(a, 1+a-b ;-1 / z) .
$$

It follows from Eq. (8) that the crossing of the threshold $S$ is an event with probability one

$$
\mathbb{P}\left(T \mid y_{0}\right)=\left(\frac{y_{0}-V_{I}}{S-V_{I}}\right)^{0} \frac{\Psi\left(0, b ; \frac{c}{y_{0}-V_{I}}\right)}{\Psi\left(0, b ; \frac{c}{S-V_{I}}\right)}=1,
$$


where we have used that $\Psi(0, b ; z)=1$ for every $z>0$. Moreover using relation (8), analogously to Ref. 54, we obtained the formula for the mean FPT

$$
\begin{aligned}
\mathbb{E}_{G}[T]= & \frac{2 \theta}{2+\theta \sigma_{G}^{2}}\left(\ln \frac{y_{0}-V_{I}}{S-V_{I}}+\left.\frac{\partial \Psi\left(a, b ; \frac{c}{S-V_{I}}\right)}{\partial a}\right|_{a=0}\right. \\
& \left.-\left.\frac{\partial \Psi\left(a, b ; \frac{c}{y_{0}-V_{I}}\right)}{\partial a}\right|_{a=0}\right)
\end{aligned}
$$

From Eq. (25), the mean FPT can be written as follows (see Appendix C):

$$
\begin{aligned}
\mathbb{E}_{G}[T]= & \frac{2 \theta}{2+\theta \sigma_{G}^{2}}\left\{\ln \frac{y_{0}-V_{I}}{S-V_{I}}-\frac{c}{b\left(y_{0}-V_{I}\right)}\right. \\
& \times{ }_{2} F_{2}\left(1,1 ; 2, b+1 ; \frac{c}{y_{0}-V_{I}}\right)+\frac{c}{b\left(S-V_{I}\right)} \\
& \times{ }_{2} F_{2}\left(1,1 ; 2, b+1 ; \frac{c}{S-V_{I}}\right)+\Gamma(b-1) \\
& \times\left[\left(\frac{c}{S-V_{I}}\right)^{1-b}{ }_{1} F_{1}\left(1-b, 2-b ; \frac{c}{S-V_{I}}\right)\right. \\
& \left.\left.-\left(\frac{c}{y_{0}-V_{I}}\right)^{1-b}{ }_{1} F_{1}\left(1-b, 2-b ; \frac{c}{y_{0}-V_{I}}\right)\right]\right\},
\end{aligned}
$$

with $b=\frac{2}{\theta \sigma_{G}^{2}}+2$ and $c=\frac{2}{\sigma_{G}^{2}}\left(\mu-\frac{V_{I}}{\theta}\right)$. The expression in Eq. (26) involves only hypergeometric functions ${ }_{p} F_{q}$ and not their derivatives and has the advantage of an easier numerical evaluation than Eq. (25).

\section{COMPARISON OF THE MODELS}

The Feller model was proved to fit experimental data of in vitro neurons under different conditions, ${ }^{58,59}$ while, to our knowledge, the IGBM model has not been applied in neuroscience. Here, we stress its existence and suggest its applicability in this context.

The parameters involved in model (1), together with the threshold $S$, can be divided into three groups: intrinsic parameters $y_{0}, V_{I}, S$; semi-intrinsic parameter $\theta$; and parameters characterizing the input $\mu, \sigma_{F}, \sigma_{G} .{ }^{60}$ In the following, we consider the same parameters values used in Refs. 30 and 41, the resetting potential is equal to zero, i.e., $y_{0}=0 \mathrm{mV}$, the inhibitory reversal potential is fixed to $V_{I}=-10 \mathrm{mV}$, and the firing threshold to $S=10 \mathrm{mV}$. The parameter of spontaneous decay is chosen $\theta=5$ or $15 \mathrm{~ms}$. Furthermore, in order to compare the models, for the choice of $\sigma_{F}$ and $\sigma_{G}$ we assume the same level of noise at the resting level, i.e., $\sigma_{F}\left(\sqrt{-V_{I}}\right)=\sigma_{G}\left(-V_{I}\right):=\sigma$.

The condition $\sigma_{F} \sqrt{-V_{I}}=\sigma_{G}\left(-V_{I}\right)$ implies that $\sigma_{F}$ $\sqrt{Y_{t}-V_{I}}>\sigma_{G}\left(Y_{t}-V_{I}\right)$ if and only if $V_{I}<Y_{t}<0$. This behavior is shown in Fig. 2 where the functions $a\left(Y_{t}\right)$ for the two processes given by Eqs. (10) and (18) are plotted in the interval $(-10,10) \mathrm{mV}$ and compared with a constant $a\left(Y_{t}\right)$ characterizing the Ornstein-Uhlenbeck process. Whereas the

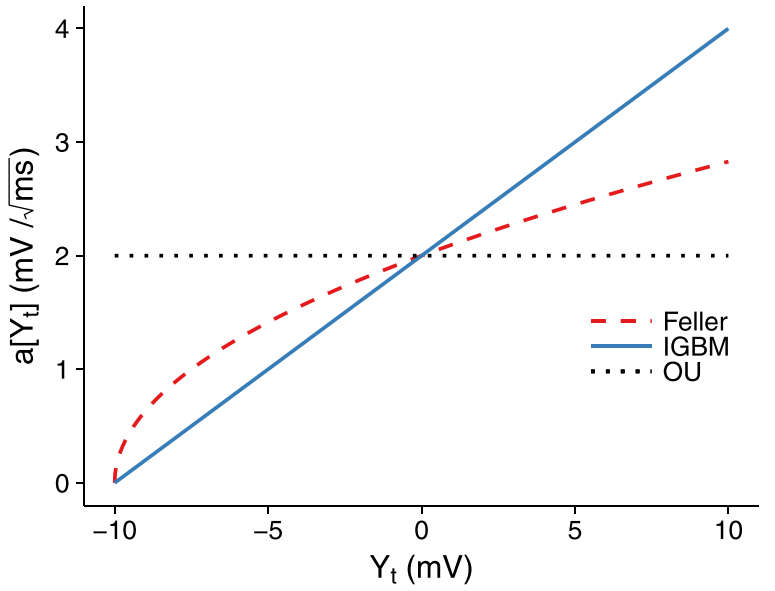

FIG. 2. Functions $a\left(Y_{t}\right)$ from Eqs. (10) and (18) in the state space $(-10,10)$ $\mathrm{mV}$, for $V_{I}=-10 \mathrm{mV}, \sigma_{F}=2 / \sqrt{10} \sqrt{\mathrm{mV}} / \sqrt{\mathrm{ms}}, \sigma_{G}=0.2 \sqrt{\mathrm{ms}}^{-1}$, and $a\left(Y_{t}\right) \equiv \sigma=2 \mathrm{mV} / \sqrt{\mathrm{ms}}$.

effect of the noise is constant for the Ornstein-Uhlenbeck model, it starts from zero at the state-space boundary and grows for the studied ones.

Finally, we always choose the values of the parameters such that the condition

$$
\mu>\frac{V_{I}}{\theta}-\frac{\sigma^{2}}{2 V_{I}}
$$

holds, which guarantees that $V_{I}$ is an entrance boundary.

The asymptotic standard deviations of the two processes are plotted as functions of the input $\mu$ for three different values of $\sigma$ in Fig. 3. The Feller process exhibits a square-root dependence, while the IGBM process exhibits a linear one, reproducing the behavior of their infinitesimal variances shown in Fig. 2. The asymptotic variability of the Feller

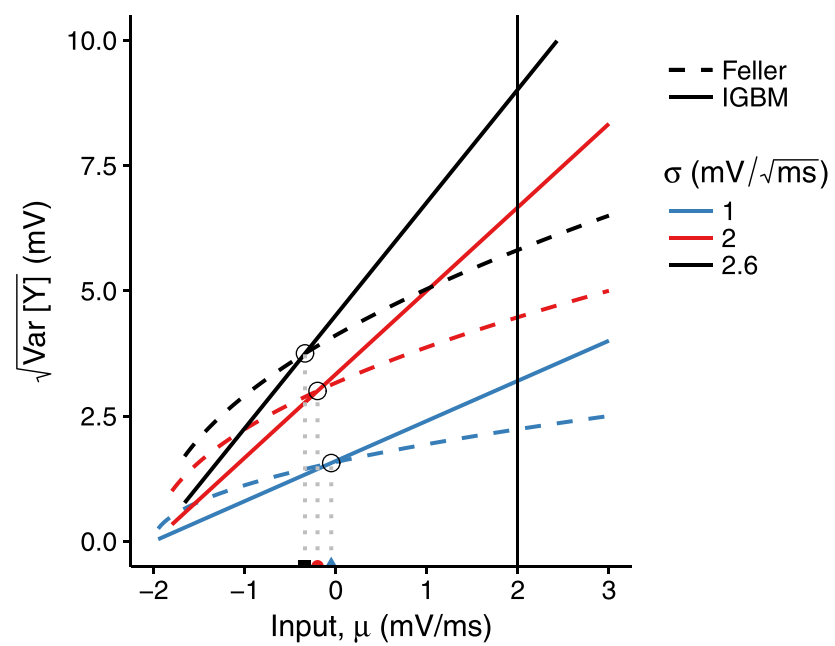

FIG. 3. Asymptotic standard deviations of $Y_{t}$ obtained from Eqs. (12) and (20) as functions of $\mu$ for $y_{0}=0 \mathrm{mV}, \theta=5 \mathrm{~ms}$, and $V_{I}=-10 \mathrm{mV}$ and for three different values of $\sigma: \sigma=1$ (blue), $\sigma=2$ (red), and $\sigma=2.6 \mathrm{mV} / \sqrt{\mathrm{ms}}$ (black). The curves intersect for $\mu=\mu^{*}$ (black circles $\mu^{*}=-0.05$, $\mu^{*}=-0.2$, and $\mu^{*}=-0.338 \mathrm{mV} / \mathrm{ms}$ ) values obtained from condition (28). For $\mu<\mu^{*}$ the Feller process exhibits higher variability than IGBM, while for $\mu>\mu^{*}$ the situation is the opposite. The vertical line separates the subthreshold $(\mu<2)$ and suprathreshold regimes $(\mu>2)$ for $S=10 \mathrm{mV}$. The smallest values of $\mu$ allowed by condition (27) are in the three cases considered: $\mu=-1.95, \mu=-1.8$, and $\mu=-1.662 \mathrm{mV} / \mathrm{ms}$. 
process is always higher than that of IGBM up to a certain input value $\mu^{*}$. More precisely, starting from Eqs. (12) and (20) one can prove that since $2 / \theta>\sigma^{2} / V_{I}^{2}$ and $\mu \theta>V_{I}$ then

$$
\operatorname{Var}_{F}[Y]>\operatorname{Var}_{G}[Y] \quad \Longleftrightarrow \mu<\mu^{*}=\frac{\sigma^{2}}{2 V_{I}} .
$$

Thus, $\mu^{*}$ decreases as $\sigma$ increases. It follows from Eq. (28) that the intersection of the two curves occurs always for $\mu<0$.

The parameter $\theta$ controls the rate at which the processes attain the asymptotic mean depolarization (5), which is the same for both of them. Looking at the slowest terms of Eqs. (11) and (19), we can have an indication of how fast the two processes reach the asymptotic variances given by Eqs. (12) and (20). We have that if

$$
\left(\frac{2}{\theta}-1\right) V_{I}^{2}<\sigma^{2}<\frac{2}{\theta} V_{I}^{2}
$$

the variability of the Feller process grows faster than that of the IGBM and all cases considered in this paper belong to this situation. Anyway, both processes reach their asymptotic variance in a relatively short time, approximately $2 \theta(10 \mathrm{~ms}$ for the case considered in Fig. 3).

We now compare the mean FPTs of Feller and IGBM processes using Eqs. (17) and (26) and we investigate how sensitive they are to a change in $\mu, \sigma$, and $\theta$. We show $\mathbb{E}_{F}[T]$ and $\mathbb{E}_{G}[T]$ as functions of $\mu$ for three different values of $\sigma$ in Fig. 4. The mean FPT decreases as $\mu$ or $\sigma$ increases for both models. For $\mu$ approaching the smallest values allowed by Eq. (27), the mean FPT tends to infinity in all considered cases. The reason is the following. For values of $\mu$ such that Eq. (27) is not fulfilled, the processes are absorbed at $V_{I}$ and consequently, they cannot cross $S$, making the FPT infinite. For a better insight, we consider the neuron to be silent (not firing) if its mean FPT is greater than $10 \mathrm{~s}$. We observe that for some values of $\sigma$, there exists a range of values of $\mu$ (colored in grey in the plots) such that the neuron described

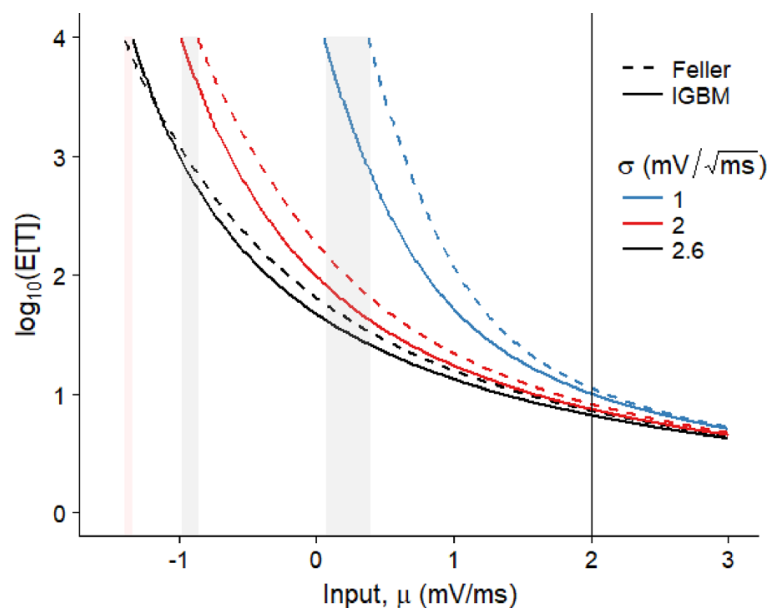

FIG. 4. Mean FPT as function of $\mu$ for Feller (dashed lines) and IGBM (solid lines) processes for $y_{0}=0 \mathrm{mV}, V_{I}=-10 \mathrm{mV}, S=10 \mathrm{mV}, \theta=5 \mathrm{~ms}, \sigma=1 ; 2$ and $2.6 \mathrm{mV} / \sqrt{\mathrm{ms}}$. The regime is subthreshold if $\mu<2 \mathrm{mV} / \mathrm{ms}$. We consider only times smaller than $10^{4} \mathrm{~ms}$ ( 4 in the log-10 scale). The range of $\mu$ such that the neuron described by the IGBM process fires in mean within $10 \mathrm{~s}$ and the one described by Feller does not is colored in grey. For $\sigma=2.6 \mathrm{mV} / \sqrt{\mathrm{ms}}$, the situation is the opposite and the corresponding range of $\mu$ is colored in red. by the Feller process is silent, while the one described by the IGBM process is not. This range becomes wider for decreasing values of $\sigma$ (see Fig. 4 for $\sigma=2 \mathrm{mV} / \sqrt{\mathrm{ms}}$ or $\sigma=1 \mathrm{mV} / \sqrt{\mathrm{ms}}$ ). For increasing values of $\sigma$ the situation starts to get reversed and the corresponding range is colored in red. In other words, both functions $\mathbb{E}_{F}[T]$ and $\mathbb{E}_{G}[T]$ grow for decreasing $\mu$ but with different speeds and this generates the gap between the mean FPTs.

To explain better this difference, the relative distance $\left|\mathbb{E}_{G}[T]-\mathbb{E}_{F}[T]\right| /\left(\mathbb{E}_{G}[T]+\mathbb{E}_{F}[T]\right)$ is plotted in Fig. 5 as a function of $\mu$ for the same values of $\sigma$ as in Fig. 4. The values of $\mu_{0}$ for which the relative distance is zero are the points of intersection in Fig. 4 (not shown for $\sigma=1$ and $2 \mathrm{mV} / \sqrt{\mathrm{ms}}$ due to the limit of $10 \mathrm{~s}$ ) and are marked by special points in Fig. 5 . For $\mu$ smaller than $\mu_{0}$, the relative distance grows quickly to 1 for all values of $\sigma$. For $\mu$ greater than $\mu_{0}$, the maximum distance between the curves decreases as $\sigma$ increases.

The asymptotic variabilities alone cannot explain all the features of the mean FPTs. First of all, the points $\mu_{0}$ and $\mu^{*}$ do not coincide; the values of $\mu_{0}$ are smaller than those of $\mu^{*}$. Moreover, the values of $\mu_{0}$ increase with $\sigma$, while the values of $\mu^{*}$ decrease (cf. colored points in Fig. 5). In contradiction with the intuitive expectation that higher variability implies shorter FPT, we see values of $\mu$ such that the mean FPT of IGBM is smaller than Feller's one although the corresponding Feller's asymptotic variance is higher (for instance compare Figs. 3 and 4 in the case $\sigma=2 \mathrm{mV} / \sqrt{\mathrm{ms}}$ and $\mu=-0.6 \mathrm{mV} / \mathrm{ms})$. The explanation can be found in the profile of the stationary distribution of $Y_{t}$ from Eqs. (13) and (21) (Fig. 6). We see that the probability that the IGBM process reaches depolarization $10 \mathrm{mV}$ or more is higher than that of the Feller process, even if the Feller process has higher asymptotic variance (top right of Fig. 6).

Finally, the role of $\theta$ is investigated showing $\mathbb{E}_{F}[T]$ and $\mathbb{E}_{G}[T]$ as functions of $\mu$ for two different values of $\theta$ in Fig. 7. For both processes, the mean FPT is bigger if $\theta$ is smaller. $\operatorname{Var}_{F}[Y]$ and $\operatorname{Var}_{G}[Y]$ are small for small $\theta$ and the processes do not move far away from the asymptotic mean

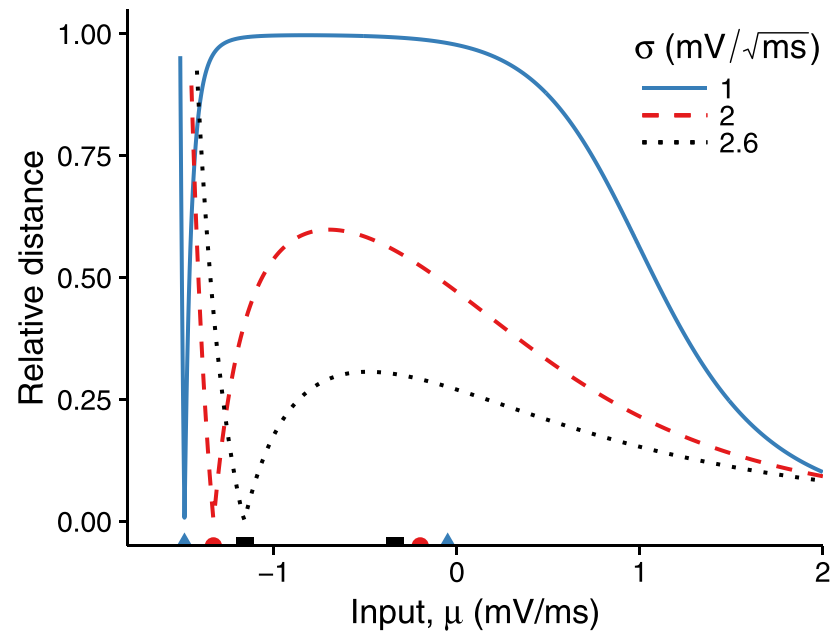

FIG. 5. Relative distance $\left|\mathbb{E}_{G}[T]-\mathbb{E}_{F}[T]\right| /\left(\mathbb{E}_{G}[T]+\mathbb{E}_{F}[T]\right)$ as a function of $\mu$ for $y_{0}=0 \mathrm{mV}, V_{I}=-10 \mathrm{mV}, S=10 \mathrm{mV}, \theta=5 \mathrm{~ms}$, and three different values of $\sigma: 2.6$ (dotted black line), 2 (dashed red line), and $\sigma=1 \mathrm{mV} / \sqrt{\mathrm{ms}}$ (solid blue line). In order to compare the values $\mu_{0}$ and the values $\mu^{*}$ obtained in Fig. 3, we use points with different shapes and colors. 


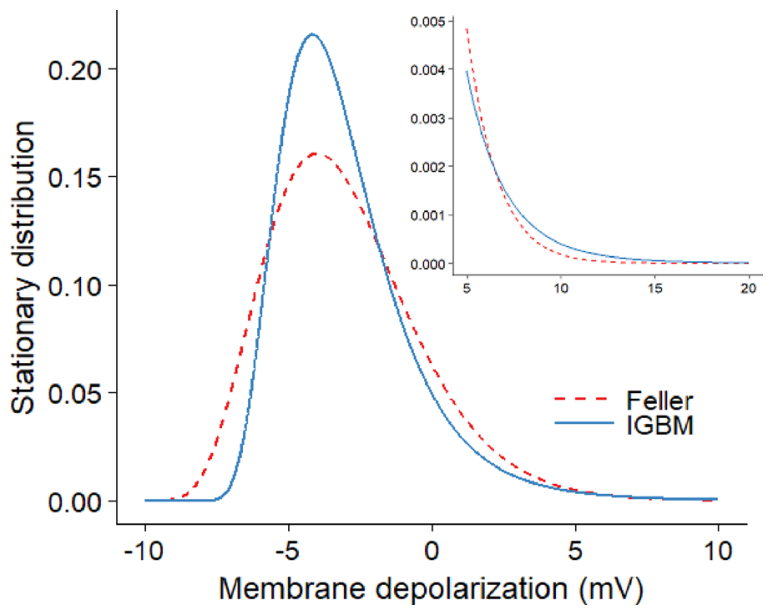

FIG. 6. The stationary distribution of $Y_{t}$ from Eqs. (13) and (21) for $y_{0}=0$ $\mathrm{mV}, V_{I}=-10 \mathrm{mV}, \sigma=2 \mathrm{mV} / \sqrt{\mathrm{ms}}, \theta=5 \mathrm{~ms}$, and $\mu=-0.6 \mathrm{mV} / \mathrm{ms}$. The processes have the same asymptotic mean $\mu \theta=-3 \mathrm{mV}$, but the Feller process has larger asymptotic variance than the IGBM process. In the zoomed plot on the top right of the figure, we see that the probability that the IGBM process reaches depolarization $10 \mathrm{mV}$ or more is higher than that of the Feller model.

level $(\mu \theta)$. This implies that if the regime is subthreshold the crossings are more rare, asymptotically for $\theta \rightarrow 0$ the mean FPT is $+\infty$. The width of the range of $\mu$ such that the Feller process is silent while the IGBM is not decreases as $\theta$ increases, similarly to what happen for increasing $\sigma$.

We conclude stressing that the formulas of the asymptotic variances of the voltage and the mean FPTs of the two models were compared with the corresponding quantities obtained via simulations, confirming the validity of the analytical expressions. To simulate Eq. (1), we used the following Milstein's scheme of discretization ${ }^{61}$

$$
\begin{aligned}
Y_{n}= & Y_{n-1}+\left(-\frac{Y_{n-1}}{\theta}+\mu\right) \Delta t+a\left(Y_{n-1}\right) \Delta W_{n-1} \\
& +\frac{1}{2} \sqrt{a\left(Y_{n-1}\right)}\left(a\left(Y_{n-1}\right)\right)^{\prime}\left[\left(\Delta W_{n-1}\right)^{2}-\Delta t\right]
\end{aligned}
$$

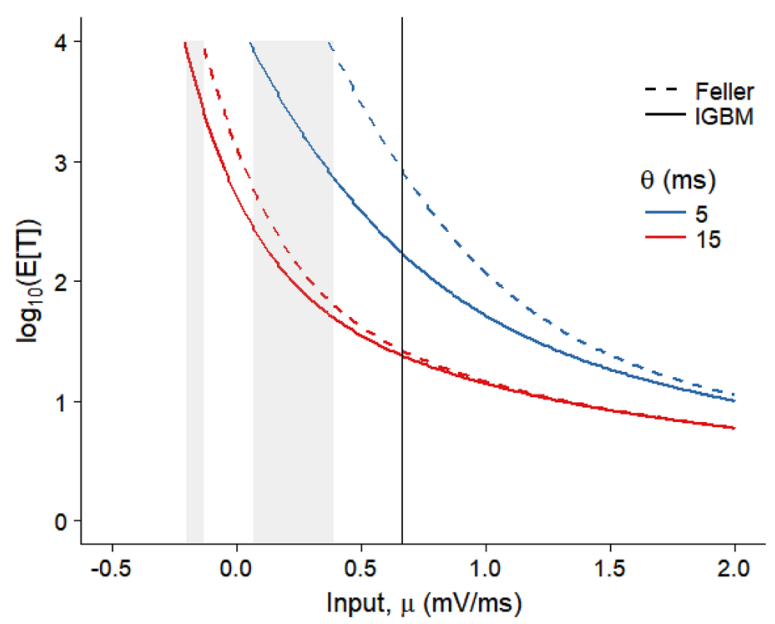

FIG. 7. Mean FPT as a function of $\mu$ for Feller (dashed lines) and IGBM (solid lines) processes for $y_{0}=0 \mathrm{mV}, V_{I}=-10 \mathrm{mV}, S=10 \mathrm{mV}, \sigma=1 \mathrm{mV} /$ $\sqrt{\mathrm{ms}}, \theta=5$ (blue lines), and $15 \mathrm{~ms}$ (red lines) in the subthreshold regime for $\theta=5 \mathrm{~ms}$, while the two regimes are separated by the black vertical line for $\theta=15 \mathrm{~ms}$. The value $b=\frac{2 V_{I}^{2}}{\theta \sigma}+2 \in \mathbb{Z}$ for $\sigma=1$ or $\sigma=2 \mathrm{mV} / \sqrt{\mathrm{ms}}$ and $\theta=5 \mathrm{~ms}$ and thus some care must be taken (see Appendix C). that is a generalization of the Euler-Marayuma discretization scheme used when the function $a(\cdot)$ depends on $Y_{t}$.

\section{CONCLUSION}

Two stochastic models describing the membrane depolarization and spike generation were compared. Both are driven by the same deterministic force but differ in the form of multiplicative noise. If the deterministic part of the model dominates, in the suprathreshold regime, both models exhibit similar behavior. The differences appear to be substantial in the subthreshold regime. There we observe that due to the different tails of the asymptotic distributions of the two processes, higher variability reflected by the second statistical moment does not always imply shorter mean FPT. The novel formulas were proposed for the precise evaluation of the mean FPT of the two processes. These expressions constitute a general result and can be used in other applications beyond that considered in this paper. Generalization to other forms of the multiplicative noise and investigation of the higher FPT moments will be the subject of our future work.

\section{ACKNOWLEDGMENTS}

This work was supported by the Institute of Physiology RVO:67985823 and by the Czech Science Foundation Project No. 17-06943S.

\section{APPENDIX A: THE EXPRESSION OF THE MEAN OF $T$ FOR THE FELLER PROCESS}

In order to use Eq. (8), we need to calculate the derivative of $g^{*}(\beta)$ given by

$$
g^{*}(\beta)=\frac{\Phi\left(a, b ; c\left(y_{0}-V_{I}\right)\right)}{\Phi\left(a, b ; c\left(S-V_{I}\right)\right)},
$$

where

$$
a=\beta \theta, \quad b=\frac{1}{2 \sigma_{F}^{2}}\left(\mu-\frac{V_{I}}{\theta}\right), \quad c=\frac{1}{2 \theta \sigma_{F}^{2}} .
$$

Since $\beta=0$ implies $a=0$ and $\Phi\left(0, b ; c\left(y_{0}-V_{I}\right)\right)=1$, then

$$
\begin{aligned}
\left.\frac{\partial g^{*}(\beta)}{\partial \beta}\right|_{\beta=0}= & \left.\left.g^{*}(\beta)\right|_{\beta=0} \frac{\partial \ln g^{*}(\beta)}{\partial \beta}\right|_{\beta=0}=\left.\frac{\partial \ln g^{*}(\beta)}{\partial \beta}\right|_{a=0} \\
= & \left(\frac{\partial \Phi\left(a, b ; c\left(y_{0}-V_{I}\right)\right)}{\partial a} \frac{\partial a}{\partial \beta}\right. \\
& \left.-\frac{\partial \Phi\left(a, b ; c\left(S-V_{I}\right)\right)}{\partial a} \frac{\partial a}{\partial \beta}\right)\left.\right|_{a=0} \\
= & \theta\left(\left.\frac{\partial \Phi\left(a, b ; c\left(y_{0}-V_{I}\right)\right)}{\partial a}\right|_{a=0}\right. \\
& \left.-\left.\frac{\partial \Phi\left(a, b ; c\left(S-V_{I}\right)\right)}{\partial a}\right|_{a=0}\right)
\end{aligned}
$$

Moreover, the derivative of the Kummer's function $\Phi(a, b ; z)$ with respect to $a$ is such that ${ }^{62}$ 


$$
\left.\frac{\partial \Phi(a, b ; z)}{\partial a}\right|_{a=0}=\frac{z}{b}{ }^{2} F_{2}(1,1 ; 2, b+1 ; z) .
$$

Equations (A2), (A3), and (8) taken together imply Eq. (17).

\section{APPENDIX B: THE LAPLACE TRANSFORM OF TFOR THE IGBM PROCESS}

The general solution of the Siegert's equation (9) with $a\left(y_{0}\right)=\sigma_{G}\left(y_{0}-V_{I}\right)$ is given by

$$
\begin{aligned}
g^{*}(\beta)= & A\left(y_{0}-V_{I}\right)^{-a} \Phi\left(a, b ; \frac{c}{y_{0}-V_{I}}\right) \\
& +B\left(y_{0}-V_{I}\right)^{-a} \Psi\left(a, b ; \frac{c}{y_{0}-V_{I}}\right),
\end{aligned}
$$

with

$$
\begin{aligned}
& a=\frac{\sqrt{\sigma_{G}^{4}+4\left(\frac{1}{\theta}+2 \beta\right) \sigma_{G}^{2}+\frac{4}{\theta^{2}}}-\left(\frac{2}{\theta}+\sigma_{G}^{2}\right)}{2 \sigma_{G}^{2}} \\
& b=\frac{2}{\theta \sigma_{G}^{2}}+2 a+2 ; \quad c=\frac{2}{\sigma_{G}^{2}}\left(\mu-\frac{V_{I}}{\theta}\right) ;
\end{aligned}
$$

and $A, B \in \mathbb{R}$. Moreover, the condition $g^{*}(\beta)<+\infty$ for any $y_{0}$ implies that $g^{*}(\beta)<+\infty$ also for $y_{0} \rightarrow V_{I}^{+}$. The term $c /\left(y_{0}-V_{I}\right) \rightarrow \infty$ for $y_{0} \rightarrow V_{I}^{+}$and since the asymptotic behaviors of $\Phi$ and $\Psi$ are such that ${ }^{63}$

$$
\begin{aligned}
\Phi(u, v ; x) & =\frac{\Gamma(v)}{\Gamma(u)} e^{x} x^{u-v}\left(1+O\left(x^{-1}\right)\right) \\
\Psi(u, v ; x) & =x^{-u}\left(1+O\left(x^{-1}\right)\right),
\end{aligned}
$$

the coefficient $A$ must be equal to zero. Finally, the condition $g^{*}(\beta)=1$ for $y_{0} \equiv S$ implies that

$$
B=\left(S-V_{I}\right)^{a} \frac{1}{\Psi\left(a, b ; \frac{c}{S-V_{I}}\right)} .
$$

Applying the above considerations on the coefficients $A$ and $B$ to Eq. (B1), one obtains Eq. (22).

\section{APPENDIX C: THE EXPRESSION OF THE MEAN OF $T$ FOR THE IGBM PROCESS}

The Tricomi's function $\Psi(a, b ; z)$ can be represented in terms of the Kummer's function $\Phi(a, b ; z)$ for $b \notin \mathbb{Z}$ and their derivatives with respect to $a$ are in the following relation: ${ }^{55}$

$$
\begin{aligned}
\left.\frac{\partial \Psi(a, b ; z)}{\partial a}\right|_{a=0}= & -\psi(1-b)+\Gamma(b-1) z^{1-b} \\
& \times \Phi(1-b, 2-b ; z)+\left.\frac{\partial \Phi(a, b ; z)}{\partial a}\right|_{a=0}
\end{aligned}
$$

where $\psi(x)=\Gamma^{\prime}(x) / \Gamma(x)$ is the digamma function. Taking together Eqs. (C1) and (A3) and the definition of Kummer's function, from Eq. (25), one obtains Eq. (26). The relation formula between $\Phi(a, b, z)$ and $\Psi(a, b, z)$ holds only for $b \notin \mathbb{Z}$; if $b \in \mathbb{Z}$, Eq. (26) is intended as taking the following limit:

$$
\Psi(a, b, z)=\lim _{\epsilon \rightarrow 0} \Psi(a, b+\epsilon, z) .
$$

${ }^{1}$ A. Bulsara, P. Hanggi, F. Marchesoni, F. Moss, and M. Shlesinger, in Proceedings of the NATO Advanced Research Workshop - Stochastic Resonance in Physics and Biology - Preface, [J. Stat. Phys. 70, 1 (1993)]. ${ }^{2}$ J. Douglass, L. Wilkens, E. Pantazelou, and F. Moss, "Noise enhancement of information-transfer in crayfish mechanoreceptors by stochastic resonance," Nature 365, 337 (1993).

${ }^{3}$ A. Longtin, "Stochastic resonance in neuron models," J. Stat. Phys. 70, 309 (1993).

${ }^{4}$ B. Lindner, J. Garca-Ojalvo, A. Neiman, and L. Schimansky-Geier, "Effects of noise in excitable systems," Phys. Rep. 392, 321 (2004).

${ }^{5}$ A. R. Bulsara, S. B. Lowen, and C. D. Rees, "Cooperative behavior in the periodically modulated Wiener process: Noise-induced complexity in a model neutron," Phys. Rev. E 49, 4989 (1994).

${ }^{6}$ B. Lindner, L. Schimansky-Geier, and A. Longtin, "Maximizing spike train coherence or incoherence in the leaky integrate-and-fire model," Phys. Rev. E 66, 031916 (2002).

${ }^{7}$ A. Longtin, B. Doiron, and A. R. Bulsara, "Noise-induced divisive gain control in neuron models," Biosystems 67, 147 (2002).

${ }^{8}$ A. Buonocore, L. Caputo, E. Pirozzi, and L. Ricciardi, "On a stochastic leaky integrate-and-fire neuronal model," Neural Comput. 22, 2558 (2010).

${ }^{9}$ G. D'Onofrio and E. Pirozzi, "Successive spike times predicted by a stochastic neuronal model with a variable input signal," Math. Biosci. Eng. 13, 495 (2016).

${ }^{10}$ I. Bashkirtseva, A. B. Neiman, and L. Ryashko, "Stochastic sensitivity analysis of noise-induced suppression of firing and giant variability of spiking in a Hodgkin-Huxley neuron model," Phys. Rev. E 91, 052920 (2015).

${ }^{11}$ S. G. Lee, A. Neiman, and S. Kim, "Coherence resonance in a HodgkinHuxley neuron,” Phys. Rev. E 57, 3292 (1998).

${ }^{12} \mathrm{C}$. Zhou and J. Kurths, "Noise-induced synchronization and coherence resonance of a Hodgkin-Huxley model of thermally sensitive neurons," Chaos 13, 401 (2003).

${ }^{13}$ M. T. Huber and H. A. Braun, "Conductance versus current noise in a neuronal model for noisy subthreshold oscillations and related spike generation," Biosystems 89, 38 (2007).

${ }^{14}$ H. A. Braun, J. Schwabedal, M. Dewald, C. Finke, S. Postnova, M. T. Huber, B. Wollweber, H. Schneider, M. C. Hirsch, K. Voigt, U. Feudel, and F. Moss, "Noise-induced precursors of tonic-to-bursting transitions in hypothalamic neurons and in a conductance-based model," Chaos 21, 047509 (2011).

${ }^{15}$ G. A. Cecchi, M. Sigman, J.-M. Alonso, L. Martnez, D. R. Chialvo, and M. O. Magnasco, "Noise in neurons is message dependent," Proc. Natl. Acad. Sci. 97, 5557 (2000).

${ }^{16}$ R. Naud, A. Payeur, and A. Longtin, "Noise gated by dendrosomatic interactions increases information transmission," Phys. Rev. X 7, 031045 (2017).

${ }^{17}$ K. Rajdl, P. Lansky, and L. Kostal, "Entropy factor for randomness quantification in neuronal data," Neural Networks 95, 57 (2017).

${ }^{18}$ M. Levakova, M. Tamborrino, L. Kostal, and P. Lansky, "Accuracy of rate coding: When shorter time window and higher spontaneous activity help," Phys. Rev. E 95, 022310 (2017).

${ }^{19} \mathrm{P}$. Dayan and L. Abbott, Theoretical Neuroscience: Computational and Mathematical Modeling of Neural Systems (Massachusetts Institute of Technology Press, 2005).

${ }^{20}$ W. Gerstner and W. Kistler, Spiking Neuron Models: An Introduction (Cambridge University Press, New York, NY, USA, 2002).

${ }^{21}$ S. Karlin and H. Taylor, A Second Course in Stochastic Processes (Academic Press, 1981), Vol. 2.

${ }^{22}$ N. Goel and N. Richter-Dyn, Stochastic Models in Biology (Academic Press, 1974).

${ }^{23} \mathrm{H}$. Tuckwell, Introduction to Theoretical Neurobiology: Volume 2, Nonlinear and Stochastic Theories (Cambridge University Press, 2005). 
${ }^{24}$ S. Shinomoto, Y. Sakai, and S. Funahashi, "The Ornstein-Uhlenbeck process does not reproduce spiking statistics of neurons in prefrontal cortex," Neural Comput. 11, 935 (1999).

${ }^{25} \mathrm{P}$. Lansky, P. Sanda, and J. He, "Effect of stimulation on the input parameters of stochastic leaky integrate-and-fire neuronal model," J. Physiol.Paris 104, 160 (2010).

${ }^{26}$ J. Inoue, S. Sato, and L. M. Ricciardi, "On the parameter estimation for diffusion models of single neuron's activities,” Biol. Cybern. 73, 209 (1995).

${ }^{27}$ H. C. Tuckwell, "Synaptic transmission in a model for stochastic neural activity,” J. Theor. Biol. 77, 65 (1979).

${ }^{28}$ P. I. M. Johannesma, "Diffusion models for the stochastic activity of neurons," in Proceedings of the -School on Neural Networks, Ravello, June 1967, edited by E. R. Caianiello (Springer Berlin Heidelberg, Berlin, Heidelberg, 1968), pp. 116-144.

${ }^{29} \mathrm{P}$. Lansky and V. Lanska, "Diffusion approximation of the neuronal model with synaptic reversal potentials," Biol. Cybern. 56, 19 (1987).

${ }^{30}$ V. Lanska, P. Lansky, and C. E. Smith, "Synaptic transmission in a diffusion model for neural activity," J. Theor. Biol. 166, 393 (1994).

${ }^{31}$ M. Musila and P. Lansky, "On the interspike intervals calculated from diffusion approximations of Stein's neuronal model with reversal potentials," J. Theor. Biol. 171, 225 (1994).

${ }^{32}$ M. J. E. Richardson and W. Gerstner, "Statistics of subthreshold neuronal voltage fluctuations due to conductance-based synaptic shot noise," Chaos 16, 026106 (2006).

${ }^{33}$ F. B. Hanson and H. C. Tuckwell, "Diffusion approximation for neuronal activity including synaptic reversal potentials,” J. Theor. Neurobiol. 2, 127 (1983).

${ }^{34}$ R. Schmidt, Fundamentals of Neurophysiology (Springer-Verlag, 1978).

${ }^{35}$ J. H. Goldwyn and E. Shea-Brown, "The what and where of adding channel noise to the Hodgkin-Huxley equations," PLOS Comput. Biol. 7, e1002247 (2011).

${ }^{36}$ J. M. Newby, P. C. Bressloff, and J. P. Keener, "Breakdown of fast-slow analysis in an excitable system with channel noise," Phys. Rev. Lett. 111, 128101 (2013).

${ }^{37}$ D. F. Anderson, B. Ermentrout, and P. J. Thomas, "Stochastic representations of ion channel kinetics and exact stochastic simulation of neuronal dynamics," J. Comput. Neurosci. 38, 67 (2015).

${ }^{38}$ D. Dufresne, The Integrated Square-Root Process (Centre for Actuarial Studies, Department of Economics, University of Melbourne, 2001).

${ }^{39}$ W. Feller, "Two singular diffusion problems," Ann. Math. 54, 173 (1951).

${ }^{40}$ S. Ditlevsen and P. Lansky, "Estimation of the input parameters in the Feller neuronal model,” Phys. Rev. E 73, 061910 (2006).

${ }^{41}$ P. Lansky, L. Sacerdote, and F. Tomassetti, "On the comparison of Feller and Ornstein-Uhlenbeck models for neural activity," Biol. Cybern. 73, 457 (1995).

${ }^{42}$ J. C. Cox, J. E. Ingersoll, and S. A. Ross, "A theory of the term structure of interest rates," Econometrica 53, 385 (1985).

${ }^{43}$ K. C. Chan, G. A. Karolyi, F. A. Longstaff, and A. B. Sanders, "An empirical comparison of alternative models of the short-term interest rate," J. Finance 47, 1209 (1992).
${ }^{44} \mathrm{O}$. Aalen and H. Gjessing, "Survival models based on the OrnsteinUhlenbeck process," Lifetime Data Anal. 10, 407 (2004).

${ }^{45}$ A. R. Pedersen, "Estimating the nitrous oxide emission rate from the soil surface by means of a diffusion model," Scand. J. Stat. 27, 385 (2000).

${ }^{46}$ C. R. Doering, K. V. Sargsyan, and P. Smereka, "A numerical method for some stochastic differential equations with multiplicative noise," Phys. Lett. A 344, 149 (2005).

${ }^{47}$ M. Brennan and E. S. Schwartz, "A continuous time approach to the pricing of bonds," J. Banking Finance 3, 133 (1979).

${ }^{48}$ G. Barone-Adesi, H. Rasmussen, and C. Ravanelli, "An option pricing formula for the GARCH diffusion model," Comput. Stat. Data Anal. 49, 287 (2005).

${ }^{49}$ R. Gutiérrez, L. Ricciardi, P. Román, and F. Torres, "First-passage-time densities for time-non-homogeneous diffusion processes," J. Appl. Probab. 34, 623 (1997).

${ }^{50} \mathrm{~V}$. Linetsky, "The spectral decomposition of the option value," Int. J. Theor. Appl. Finance 7, 337 (2004).

${ }^{51} \mathrm{M}$. Insley, "A real options approach to the valuation of a forestry investment," J. Environ. Econ. Manage. 44, 471 (2002).

${ }^{52}$ S. Sarkar, "The effect of mean reversion on investment under uncertainty," J. Econ. Dyn. Control 28, 377 (2003).

${ }^{53}$ L. M. Abadie and J. M. Chamorro, "Valuing flexibility: The case of an integrated gasification combined cycle power plant," Energy Econ. 30, 1850 (2008).

${ }^{54}$ B. Zhao, "Inhomogeneous geometric Brownian motions," SSRN Electron. J. 38 (2009).

${ }^{55}$ M. Abramowitz and I. Stegun, Handbook of Mathematical Functions: With Formulas, Graphs, and Mathematical Tables (Dover Publications, 1964).

${ }^{56}$ V. Giorno, P. Lansky, A. Nobile, and L. Ricciardi, "Diffusion approximation and first-passage-time problem for a model neuron," Biol. Cybern. 58, 387 (1988).

${ }^{57}$ R. K. S. Hankin, Hypergeo: The Gauss Hypergeometric Function, $R$ Package Version 1.2-13 (2016); available at https://CRAN.R-project.org/ package=hypergeo.

${ }^{58}$ P. Jahn, R. W. Berg, J. Hounsgaard, and S. Ditlevsen, "Motoneuron membrane potentials follow a time inhomogeneous jump diffusion process," J. Comput. Neurosci. 31, 563 (2011).

${ }^{59} \mathrm{R}$. Hopfner, "On a set of data for the membrane potential in a neuron," Math. Biosci. 207, 275 (2007).

${ }^{60} \mathrm{H}$. C. Tuckwell and W. Richter, "Neuronal interspike time distributions and the estimation of neurophysiological and neuroanatomical parameters," J. Theor. Biol. 71(2), 167 (1978).

${ }^{61}$ P. Kloeden and E. Platen, Numerical Solution of Stochastic Differential Equations, Stochastic Modelling and Applied Probability (Springer, Berlin, Heidelberg, 2011).

${ }^{62}$ L. U. Ancarani and G. Gasaneo, "Derivatives of any order of the confluent hypergeometric function $\mathrm{f} 11(\mathrm{a}, \mathrm{b}, \mathrm{z})$ with respect to the parameter a or $\mathrm{b}$," J. Math. Phys. 49, 063508 (2008).

${ }^{63}$ Y. Luke, The Special Functions and Their Approximations (Elsevier Science, 1969). 